\title{
CORRECTION
}

\section{Correction to: Simplified methods of the 3D-SVPWM for four-wire three-leg inverter}

\author{
Fathy H. Awad ${ }^{1}$ - . Ahmed A. Mansour ${ }^{1} \cdot$ Mostafa I. Marei $^{2} \cdot$ Ahmed A. El-Sattar $^{2}$
}

Published online: 11 November 2020

(c) The Korean Institute of Power Electronics 2020

\section{Correction to: Journal of Power Electronics} https://doi.org/10.1007/s43236-020-00133-4

$$
d_{0 i=(1-48)}=M \times\left(G_{i}+H_{i} \times \theta\right) \quad \text { for } \theta=\left(0^{\circ}-120^{\circ}\right) \text {. }
$$

Due to an unfortunate oversight some equations (4, 5, 6 and

7) have been given erroneously, such that:

$\theta=\left(0^{\circ}-20^{\circ}\right)$ should be replaced by $\theta=\left(0^{\circ}-120^{\circ}\right)$ in the four equations $4,5,6,7$.

$d_{1 i=(1-48)}=M \times\left(A_{i}+B_{i} \times \theta\right)$ for $\theta=\left(0^{\circ}-120^{\circ}\right)$,

$d_{2 i=(1-48)}=M \times\left(C_{i}+D_{i} \times \theta\right) \quad$ for $\theta=\left(0^{\circ}-120^{\circ}\right)$

$d_{3 i=(1-48)}=M \times\left(E_{i}+F_{i} \times \theta\right)$ for $\theta=\left(0^{\circ}-120^{\circ}\right)$,

The original article can be found online at https://doi.org/10.1007/ s43236-020-00133-4.

Fathy H. Awad

fathyhamed@eri.sci.eg

1 Power Electronics and Energy Conversion Department, Electronics Research Institute, Cairo, Egypt

2 Electrical Power and Machine Department, Faculty of Engineering, Ain Shams University, Cairo, Egypt 